\title{
E-Learning als Möglichkeit zur Unterstützung des selbstgesteuerten Lernens an Berufsschulen'
}

\author{
Silvia Dreer
}

\begin{abstract}
Im Mittelpunkt dieses Beitrages steht die Frage, wie durch die Potenziale von ELearning selbstgesteuertes Lernen an berufsbildenden Schulen (Berufsschulen) unterstützt werden kann und wie diese neuen Lernangebote im Unterricht effektiv genutzt werden können. Dann werden bereits vorhandene empirische Belege zum selbstgesteuerten Lernen aufgearbeitet und unterschiedliche Möglichkeiten zur Förderung des selbstgesteuerten Lernens betrachtet bzw. welche Lernumgebungen notwendig sind, um selbstgesteuertes Lernen zu ermöglichen. Es wird auf die Besonderheiten von E-Learning eingegangen und eine mediendidaktische Konzeption realisiert. Im Rahmen der empirischen Untersuchung wird untersucht, ob selbstgesteuertes Lernen durch E-Learning unterstützt werden kann. Den Abschluss bildet eine Abschätzung des mediendidaktischen Potenzials von Lernumgebungen die selbstgesteuertes Lernen unterstützen in Hinblick auf den Unterricht in berufsbildenden Schulen.
\end{abstract}

\section{Einführung}

«Der Begriff der Selbststeuerung beim Lernen enthält ein anschauliches Bild: Der Lernende sitzt selbst am Steuer und fährt sein (Lern-) Auto selber, kein anderer tut dies [...]. Und da fangen die Probleme an: Er kennt nicht einmal die wichtigsten Begriffe (verwechselt Lenkrad und Steuerrad). Kann er überhaupt selber steuern? Beherrscht er die notwendigen Techniken, die Regeln, weiss er überhaupt, wohin er will? Kennt er Ziele, Wege, beherrscht er die nötigen Fertigkeiten zum Selbststeuern, kann er mit den Ressourcen (〈Treibstoff`) sinnvoll umgehen [...] ist er sich überhaupt sicher, dass ihn nicht eine fremdgesteuerte Fahrt viel schneller und sicherer ans Ziel gebracht hätte?» (Gudjons, 2003)

Dieses Zitat kann im Rahmen der Unterrichtspraxis folgendermassen interpretiert werden: Es kann nicht vorausgesetzt werden, dass Lernende automatisch «selbstgesteuert Lernen» können, sondern es müssen, bestimmte Faktoren (beispielsweise der Erwerb von Selbstlernkompetenzen) berücksichtigt werden, damit der

Dreer, Silvia. «E-Learning als Ansatz einer individualisierten Lernstrategie an einer Berufsschule für Informationstechnik.» Dissertation, Duisburg-Essen: Universität, Fachbereich Bildungswissenschaften genehmigte Dissertation, Referent: Michael Kerres, Koreferent: Joachim Rottmann, Rigorosum 20.06.2008. 
Einsatz vom selbstgesteuerten Lernen für Lernende eine positive Auswirkung auf das Lernverhalten hat. Dabei stellt sich die Frage, ob selbstgesteuertes Lernen überhaupt durch den Einsatz von E-Learning unterstützt werden kann.

\section{Potenziale von E-Learning}

Durch den Einsatz von E-Learning werden Möglichkeiten geboten, die herkömmliche Unterrichtsmethoden noch zusätzlich fördern können. Auch beim Einsatz von E-Learning im Unterricht sind bestimmte Selbstlernkompetenzen notwendig, die der Lernende erwerben oder mitbringen muss. Durch das zeit- und ortsunabhängige Lernen und die individuelle Bestimmung der Lerngeschwindigkeit durch den Lernenden können Lernprozesse individueller gestaltet werden. Es werden verschiedene Kommunikationsmöglichkeiten angeboten (z. B. Chat, E-Mail, usw.) und elektronische Lernmaterialien zur Verfügung gestellt, die der Lernende je nach Bedarf einsetzen kann. Die Lernerfolgskontrolle kann durch den Lernenden selbst erfolgen. Es wird individualisiertes und kooperatives Lernen unterstützt, wenn die didaktische Konzeption auf diese Ziele abgestimmt ist. Im Bereich der Schulen wird E-Learning bereits eingesetzt und die Verwendung dieses Werkzeuges durch den/ die Lehrer/in im Unterricht wird auch durch das Bundesministerium entsprechend gefördert. Kritisch betrachtet ist eine sorgfältige Planung und Durchführung der mediendidaktischen Konzeption erforderlich, um negative Effekte zu minimieren.

\section{Selbstgesteuertes Lernen}

Vergleicht man die in der Literatur verfügbaren Modelle zum selbstgesteuerten Lernen (Weinstein, 1994; Friedrich \& Mandl, 1997; Boakerts, 1999; Zimmerman, 2000; Pintrich, 2000; Borkowski et. al, 2000), beinhalten die betrachteten Modelle des selbstgesteuerten Lernens sowohl motivationale, kognitive (Lernstrategieeinsatz) und in den neueren Studien auch metakognitive Komponenten (Steuerung und Kontrolle des Lernprozesses), die untereinander in wechselseitiger Beziehung stehen. Die Modelle wurden aus unterschiedlichen Perspektiven betrachtet, jedoch wurde übereinstimmend festgestellt, dass das selbstgesteuerte Lernen ein aktiver und konstruktiver Wissenserwerbsprozess beim Lernenden ist. Dieser kann seine Bedürfnisse des Lernens - wie beispielsweise die Motivation, individuelle Lernziele und adäquate Lernstrategien - beim Lernprozess einsetzen. Aufgrund des starken Praxis- und Handlungsbezuges und die Anwendung im Kontext des Unterrichts würde sich das Modell von Pintrich (2000) für die vorliegende Untersuchung besonders eignen, da dieses an bereits verfügbare Modelle anknüpft und ein vollständiges Modell darstellt, erweitert um Komponenten von Borkowski (2000), in der die selbstgesteuerte Lernumgebung den Lernenden die Möglichkeit zur Förderung von Lernstrategien anbieten muss: 


\begin{tabular}{|c|c|c|c|c|}
\hline \multirow[b]{2}{*}{ Phasen } & \multicolumn{4}{|c|}{ Bereiche der Steuerung } \\
\hline & Kognition & Motivation / Affekt & Verhalten & Kontext \\
\hline $\begin{array}{l}\text { 1. Voraussicht, } \\
\text { Planung und } \\
\text { Aktivierung }\end{array}$ & $\begin{array}{l}\text { Zielsetzung, Aktivie- } \\
\text { rung von Vorwissen } \\
\text { und metakognitiven } \\
\text { Wissen }\end{array}$ & $\begin{array}{l}\text { Verfolgung einer } \\
\text { Zielorientierung, } \\
\text { Wirksamkeitsein- } \\
\text { schätzung, } \\
\text { Schwierigkeits- } \\
\text { einschätzung, Aktivie- } \\
\text { rung von Interesse }\end{array}$ & $\begin{array}{l}\text { Zeit- und Auf- } \\
\text { wandsplanung, } \\
\text { Planen von } \\
\text { Selbstbeobacht- } \\
\text { ungen }\end{array}$ & $\begin{array}{l}\text { Wahrnehmung } \\
\text { einer Aufgabe, } \\
\text { Wahrnehmung } \\
\text { eines Kon- } \\
\text { textes }\end{array}$ \\
\hline 2. Steuerung & $\begin{array}{l}\text { Aufmerksamkeit } \\
\text { und Beobachtung } \\
\text { von Kognitionen }\end{array}$ & $\begin{array}{l}\text { Aufmerksamkeit und } \\
\text { Beobachtung von Mo- } \\
\text { tivation und Affekt }\end{array}$ & $\begin{array}{l}\text { Aufmerksamkeit } \\
\text { und Beobachtung } \\
\text { von Anstrengung, } \\
\text { Zeitnutzung und } \\
\text { Notwendgeit von } \\
\text { Hilfe, Verhaltensbe- } \\
\text { obachtung }\end{array}$ & $\begin{array}{l}\text { Beobachtung } \\
\text { von Ände- } \\
\text { rungen in den } \\
\text { Aufgaben und } \\
\text { Kontextbeding- } \\
\text { ungen }\end{array}$ \\
\hline 3. Kontrolle & $\begin{array}{l}\text { Auswahl und An- } \\
\text { passung von Lern- } \\
\text { strategien }\end{array}$ & $\begin{array}{l}\text { Auswahl und Anpas- } \\
\text { sung von Strategien } \\
\text { zur Beeinflussung von } \\
\text { Motivation }\end{array}$ & $\begin{array}{l}\text { Erhöhung oder } \\
\text { Senkung von An- } \\
\text { strengung, Weiter- } \\
\text { machen oder Auf- } \\
\text { hören, Hilfesuche }\end{array}$ & $\begin{array}{l}\text { Veränderung } \\
\text { der Aufgabe, } \\
\text { Veränderung } \\
\text { oder Verlassen } \\
\text { des Kontextes }\end{array}$ \\
\hline $\begin{array}{l}\text { 4. Reaktion und } \\
\text { Reflexion }\end{array}$ & $\begin{array}{l}\text { Kognitive Bewer- } \\
\text { tung, Ursachenzu- } \\
\text { schreibungen }\end{array}$ & $\begin{array}{l}\text { Affektive Reaktionen, } \\
\text { Ursachenzuschreib- } \\
\text { ungen }\end{array}$ & Wahl von Verhalten & $\begin{array}{l}\text { Bewertung } \\
\text { der Aufgabe, } \\
\text { Bewertung des } \\
\text { Kontextes }\end{array}$ \\
\hline
\end{tabular}

Tabelle 1: Modell des selbstregulierten Lernens (Pintrich, 2000)

Alle neueren Modelle (ab 2000) beinhalten neben den motivationalen und kognitiven Komponenten auch metakognitive Komponenten, die auch als wichtig erachtet werden.

Stellt man die Modelle selbstgesteuerten Lernens gegenüber, ergeben sich folgende Gemeinsamkeiten, die insbesondere für den Einsatz des selbstgesteuerten Lernens im Bereich der berufsbildenden Schulen relevant sind:

- Motivationale Komponenten: Alle Modelle haben gemeinsam, dass motivationale Komponenten eine Grundvoraussetzung für den erfolgreichen Einsatz des selbstgesteuerten Lernens sind. Lernende müssen sich immer wieder neu selbst motivieren können, um erfolgreich Lernaufgaben zu bearbeiten.

- Kognitive Komponenten: Als weiteren wichtigen Faktor haben auch alle Modelle gemeinsam, dass die Anwendung von kognitiven Komponenten, die eine Informationsverarbeitung bei den Lernenden stattfinden lässt, als wesentlicher 
Faktor für den erfolgreichen Einsatz des selbstgesteuerten Lernens zu bewerten ist.

- Metakognitive Komponenten: Insbesondere die neueren Studien (Vgl. auch Tiaden, 2006; Pintrich, 2000; Zimmermann, 2000) und speziell die Studie von Borkowski (2000) haben sich sehr stark auf die metakognitiven Komponenten fokussiert, die für erfolgreiches selbstgesteuertes Lernen wichtig sind.

- Kontrollstrategien (Volitionale Strategien): Volitionale Bewältigungsstrategien sind für das selbstgesteuerte Lernen relevant, wie beispielsweise die Kontrolle der Aufmerksamkeit, der Motivation und die Kontrolle der Emotionen bei Misserfolgserlebnissen und sollen den selbstgesteuerten Lernprozess vor Aktivitäten schützen, die nicht mit dem Lernprozess in Zusammenhang stehen. (Vgl. Siebert, 2001)

- Einbindung in den Unterricht bzw. in eine offene Lernumgebung: Während eines Lernprozesses sollen bewährte Unterrichtsmethoden umgesetzt werden, damit der Einsatz des selbstgesteuerten Lernens erfolgreich ist. Diese Unterrichtsmethoden beziehen sich auf die konkrete Umsetzung der vorgestellten Theorien in die Unterrichtspraxis, wie beispielsweise den Lernenden Lernstrategien zu vermitteln.

- Zielsetzung durch den Lernenden: Lernende sollen entweder selbst ihre Lernziele setzen können oder eines auswählen können, das sie dann bearbeiten und die damit verbundenen weiteren Lernschritte selbst planen können.

- Lernende: Selbstlernkompetenzen (z. B. die Anwendung von Lernstrategien) sollen für unterschiedliche Lernaufgaben vorhanden oder vermittelt werden. Diese Vermittlung nimmt sehr viel Unterrichtszeit in Anspruch, die einerseits für Fachinhalte verloren geht und andererseits dafür die Lernenden wertvolle Kompetenzen für lebenslanges Lernen erwerben können, welches ein sehr wichtiges Ziel an berufsbildenden Schulen ist.

Es gibt kein für alle Anwendungskontexte geeignetes "Standardmodell» das überall gleich erfolgreich eingesetzt werden kann, da viele Forschungsergebnisse unzureichend bzw. zu wenig spezifisch sind. Problematisch ist auch, dass es zu wenige Erkenntnisse über die Lernprozesse und die Bedürfnisse des Lernenden gibt. Fehlend ist weiters ein Modell das die Annahme unterstützt, dass Lernziele auch persönliche Lernziele des Lernenden sind. Weiters sind aktuelle Theorien unvollständig, da es keine Annahmen gibt, wie Lernende mit Zielkonflikten umgehen. Selbstgesteuertes Lernen benötigt sehr viele Ressourcen und Schüler/innen verfügen nicht über Kompetenzen, vorhandene Ressourcen (Zeit, Aufmerksamkeit, etc.), so auf ihre Lernprozesse aufzuteilen, dass es optimal ist und es hier auch in der vorhandenen wissenschaftlichen Literatur keine theoretischen Modelle gibt, wie das optimal umgesetzt werden könnte. (Vgl. auch Boekaerts, 1999 \& Astleitner et al, 2003) 
In den vorgestellten Modellen gibt es für einige Bereiche zu wenige Anknüpfungen für die Einsatzmöglichkeiten in einer offenen Lernumgebung, die aber gerade im Bereich der berufsbildenden Schulen von grossem Interesse sind:

- Einsatz von selbstgesteuertem Lernen an berufsbildenden Schulen

- Lernstrategien und Zeitplanung

- Motivation

- Lernziele

- Offene Lernumgebung

- Selbstwirksamkeit

- Lernstil-Typen

Hier wurden relevante empirische Forschungsergebnisse gesammelt, die im nächsten Abschnitt «Selbstgesteuertes Lernen an berufsbildenden Schulen» noch genauer erläutert werden.

\section{Selbstgesteuertes Lernen an berufsbildenden Schulen}

Verschiedene Studien (Dumke \& Wolff-Kolmar, 1997; Ablard \& Lipschultz, 1998; Perry, 1998; Schraw, 1998; Wuttke, 1999; Hamman et al, 2000; Wild, 2000; Aeppli, 2005; Metzger et al, 2005; Salchegger, 2005; van Den Hurk, 2006; Dreyer, 2007; Stenger, 2007) zum selbstgesteuerten Lernen bestätigen, dass unterschiedliche Faktoren beim erfolgreichen Einsatz von selbstgesteuerten Lernen an berufsbildenden Schulen relevant sind. Die Rolle des Lehrers verändert sich zum Lernberater, der den Schülern Hilfestellung und Unterstützung anbietet. Weiters achtet er darauf, dass im Unterricht ausreichende Lernressourcen zur Verfügung stehen. Als Sozialform eignet sich Einzel-, Partner- oder Gruppenarbeit gleichermassen. Wenn ein Lernender ein gutes Zeitmanagement (= ressourcenbezogene Lernstrategie) hat und, je nach Lernaufgabe, geeignete unterschiedliche Lernstrategien anwenden kann, wird dieser einen hohen Lernerfolg haben. Für die Zielgruppe der schwächeren Schüler/innen, die vermutlich wenige Lernstrategien einsetzen und ihre Zeit auch wenig effektiv nutzen, sollte man ein Lernstrategietraining anbieten, damit auch diese Zielgruppe das selbstgesteuerte Lernen erfolgreich anwenden kann. Es ist darauf zu achten, dass nicht zu viele Lernstrategien auf einmal vermittelt werden, um die Lernenden nicht zu überfordern. Der Lernende soll sich selbst Ziele setzen können, die sich motivierend auf ein Fachgebiet auswirken können.

\section{Lernstrategien}

Eines der wichtigsten Bereiche beim selbstgesteuerten Lernen ist, wie bereits erwähnt, die Fähigkeit der Lernenden, aufgaben- und situationsbezogene, adäquate Lernstrategien auszuwählen, kombinieren und einzusetzen. Derartige Lernstrategien umfassen beispielsweise das Auswählen und Organisieren von Informationen, 
Anknüpfung der neuen Lerninhalte mit dem Vorwissen und auch das Wiederholen und Memorieren von Lerninhalten, die im Langzeitgedächtnis gespeichert werden sollen. Die Kenntnis und Anwendung unterschiedlicher Lernstrategien ist eine notwendige Vorraussetzung für die Kontrolle des Lernprozesses (Vgl. Baumert, 2003, Zimmerman \& Schunk, 2001)

\section{Herausforderungen in Berufsschulen}

Im Bereich der Berufsschulen gibt es für Lehrende und Lernende besondere $\mathrm{He}$ rausforderungen im Unterricht, die durch folgende Merkmale charakterisiert sind:

- Heterogenität der Berufsschüler/-innen (Einstiegsvoraussetzung für den Lehrberuf: Bandbreite von Sonderschule bzw. kein Hauptschulabschluss, Hauptschulabschluss, Reifeprüfung bis zu Studienabbrechern)

- Unterschiedliche bzw. keine berufsbezogenen Vorkenntnisse.

- Doppellehrberufe und Anrechnung von Fachklassen.

- Lehre ohne Ausbildungsbetrieb.

In Berufsschulen wird vorwiegend in den theoretischen Unterrichtsgegenständen Frontalunterricht praktiziert. Lern- und Arbeitstechniken sind bei Berufsschülern noch verbesserungswürdig. Durch diese Herausforderungen ist es notwendig, den Schülern die Befähigung zum lebenslangen Lernen durch die Förderung des selbstgesteuerten Lernens zu vermitteln und den damit verbundenen Erwerb von Selbstlern- und Medienkompetenzen im EDV-Unterricht durch Nutzung der neuen Informations- und Kommunikationstechnologien. Zusätzlich kann man den Lernenden noch eine Individualisierung im Unterricht ermöglichen, damit sich Lernende unterschiedliche Schwerpunkte setzen können. Diese Ziele können insbesondere durch den zusätzlichen Einsatz neuer Methoden im Unterricht, wie beispielsweise E-Learning, erreicht werden. (Vgl. Hasenpflug et. al, 2004, S. 6)

Die vorliegende Untersuchung will diese Herausforderungen im Umfeld der Lehrberufe IT-Technik und IT-Informatik aufgreifen und zur Verbesserung des Lehrens und Lernens an Berufsschulen beitragen. Damit verbunden wurden (kostenlos zugängliche) elektronische Lernmodule (siehe www.e-learnit.at.tf - Projekt E-LEARNIT) konzipiert, die selbstgesteuerte Lernprozesse bei Lernenden ermöglichen sollen. Lehrende verändern ihre Rolle vom Wissensvermittler zum Betreuer, der Lernprozesse begleitet, die Lernenden unterstützt, bei Fragen und Unklarheiten weiterhilft, Lernmodule produziert bzw. evaluiert und den Lernenden eine Rückmeldung zu ihren Lernergebnissen gibt. 


\section{Lernumgebung EDV-Labor in Berufsschulen}

Der berufs- und praxisbezogene Unterricht im Gegenstand EDV-Labor bietet viele Möglichkeiten, die im herkömmlichen theoretischen Unterricht nicht in dieser Form vorhanden sind: kleinere Schülergruppen, PC-Arbeitsplätze und Lernprozesse, die mehrere Stunden durchgehend andauern können. Dadurch können komplexere Lernaufgaben bearbeitet werden und theoretisches Wissen vertieft werden. Didaktische Grundsätze, dass Schüler/innen Vorwissen und Erfahrungen aus der Berufspraxis einbringen können, werden in besonderer Weise unterstützt. Kompetenzen können durch die Kommunikation bei Partner- bzw. Gruppenaufgaben, die Fähigkeit Wissen selbständig zu erarbeiten und durch den Wechsel von Individual- und Sozialphasen gefördert werden. Schüler/innen können Problemlösungsstrategien entwickeln durch den Erwerb von grundsätzlichen Herangehensweisen an praxisbezogenen EDV-Problemen.

\section{Untersuchungsgegenstand Projekt E-LEARNIT an Berufsschulen}

Das Projekt ELEARN-IT wurde von der Autorin dieses Beitrages initiiert im Rahmen des Einsatzes von E-Learning im Unterricht der Lehrberufe der Informationstechnologien (ELEARN-IT = ELEARNING INFORMATIONSTECHNOLOGIEN) und wurde ausschliesslich aus privaten finanziellen Mitteln finanziert. Als Lernplattform für dieses Projekt wurde die Open-Source Lernplattform Moodle verwendet. (Vgl. BMBWK, 2006)

Der Schwerpunkt dieser ELEARN-IT Lernumgebung liegt bei der Entwicklung eines medialen Lernangebotes, das eine Individualisierung im Rahmen des selbstgesteuerten Lernens ermöglichen soll. Durch die Einsatzmöglichkeiten der Lernplattform sollen Lernende bei unterschiedlichen Lernangeboten zum gleichen Themenkreis eine Wahlmöglichkeit bei den inhaltlichen Schwerpunkten finden. Es sollen im Unterricht individuelle Schwerpunkte durch den Lernenden gesetzt werden können, in jeder E-Learning Lerneinheit eine freie Themenwahl stattfinden (Zeit- und Contentfahrplan) und unterschiedliche Lerntypen berücksichtigt werden. 
Homepage zum Projekt ELEARN-IT online im Internet:

URL: http://www.e-learnit.at.tf [Stand: 20.04.2008]

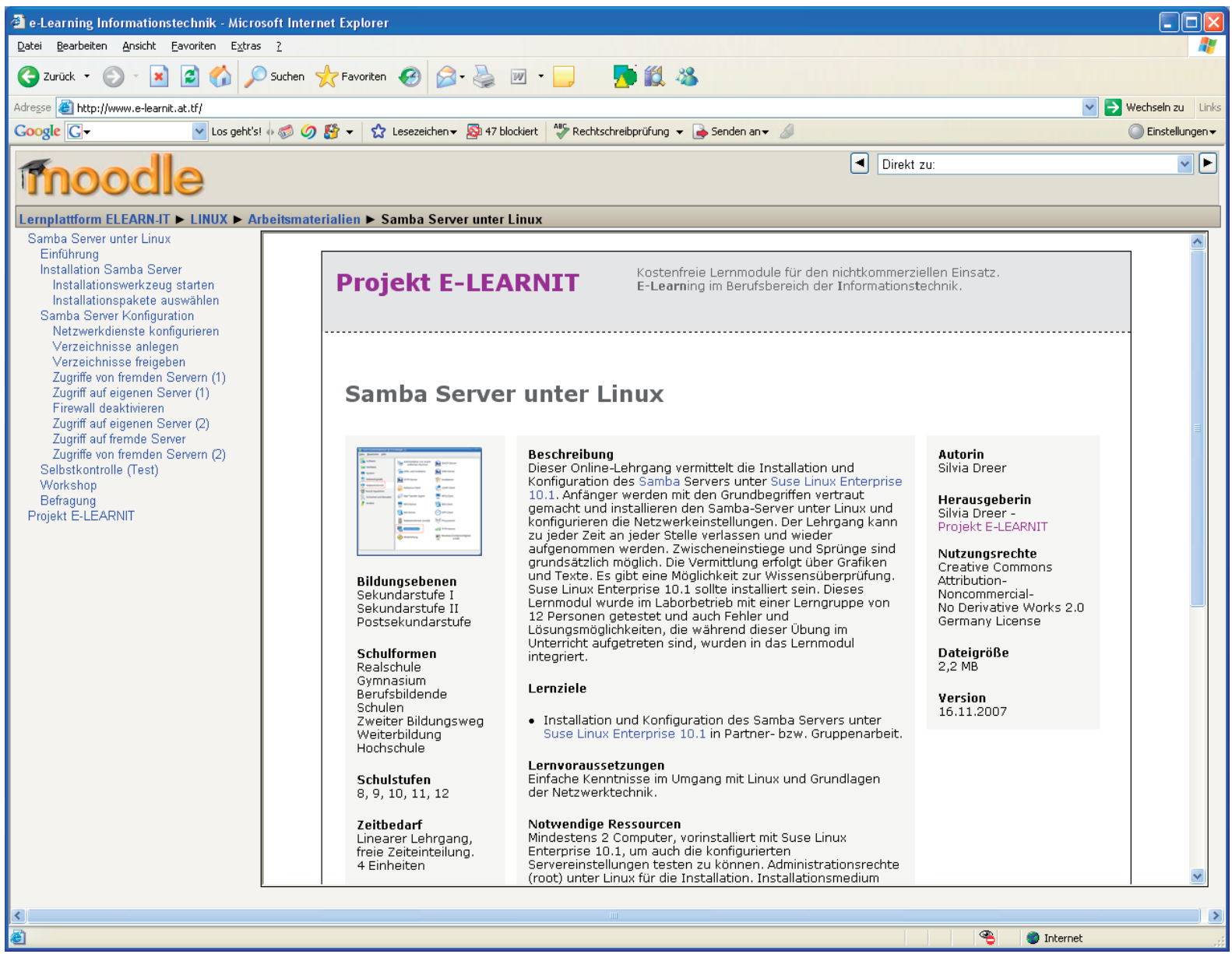

Abbildung 1: Lernplattform ELEARN-IT, Lernmodul Samba Server unter Linux

\section{«Zeit- und Contentfahrplan» Curriculumentwicklung}

Durch den «Zeit- und Contentfahrplan» (Vgl. Kerres \& Petschenka, 2002), der den Schülern zu Unterrichtsbeginn ausgehändigt wird, können diese aus unterschiedlichen Themen, Schwierigkeitsstufen und Sozialformen individuell auswählen. Die in einer Lernaufgabe erreichten Punkte werden durch den/die Betreuer/in an den/ die Schüler/in innerhalb von 5 Tagen nach der Abgabe der (individuellen) Lösung bekannt gegeben, weshalb eine Transparenz gewährleistet wird. Idealerweise gibt so ein Arbeitsplan die Themen, Aufgaben, Schwierigkeitsstufen und Lernziele vor und bietet dennoch Freiräume für individuelle Schwerpunkte und Interessen. (Vgl. Bräu, 2005) 


\begin{tabular}{|c|c|c|c|c|c|c|}
\hline \multicolumn{7}{|c|}{ Schwierigkeitsstufe S (sehr einfach) } \\
\hline Datum & \begin{tabular}{|l} 
Zeitdauer \\
(UE)
\end{tabular} & Sozialform & Thema & Schwierigeit & $\begin{array}{l}\text { Maximale } \\
\text { Punkte }\end{array}$ & $\begin{array}{l}\text { Erreichite } \\
\text { Punkte }\end{array}$ \\
\hline 20.09 .2007 & 2 & Einzelarbeit & Firewall - Zone Alarm & 5 & 10 & 7 \\
\hline 27.09 .2007 & 2 & Partner: Bart Simpson & Windows Firemall & 5 & 10 & 1 \\
\hline 5.10 .2007 & 2 & Einzelarbeit & Linux Installation & s & 10 & 9 \\
\hline 30.10 .2007 & 3 & Einzelarbeit & Linux Grundlagen & 5 & 10 & 10 \\
\hline 5.11 .2007 & 2 & Gruppe: Lisa + Bart Simpson & Linux Befente & 5 & 10 & 10 \\
\hline 12.11 .2007 & 4 & Einzelarbeit & Apache Webserver unter Linux & s & 10 & 10 \\
\hline 14.11 .2007 & 3,5 & Einzelarbeit & Google - Suche & 5 & 10 & 10 \\
\hline 19.11 .2007 & 6 & Einzelarbeit & WPNiNetzwerk & s & 10 & 5 \\
\hline 20.11 .2007 & 3 & Einzelarbeit & Netzlwerk-Sniffer & s & 10 & 10 \\
\hline 30.11 .2007 & 2 & Einzelarbeit & Netzwerkanalyse & s & 10 & 10 \\
\hline \multicolumn{5}{|c|}{ SUMIFE Stufe S } & max. 100 Punke & 87 Punkte \\
\hline \multicolumn{7}{|c|}{ Schwierigkeitsstufe M (mittelmässig) } \\
\hline Datum & \begin{tabular}{|l} 
Zeitdauer \\
(UE)
\end{tabular} & Sozialform & Thema & Schusierigkeit & $\begin{array}{l}\text { Maximale } \\
\text { Punkte }\end{array}$ & $\begin{array}{l}\text { Erreichite } \\
\text { Punkte }\end{array}$ \\
\hline 20.09 .2007 & 2 & Partner: Marge Simpson & Dws server unter Linux & Wh & 10 & 10 \\
\hline $27,09.2007$ & 4 & Einzelarbeit & Samba Server unter Linux & m & 10 & 9 \\
\hline 5.10 .2007 & 3 & Einzelarbeit & Windows 2003 Server - Grundlagen & m & 10 & 10 \\
\hline \multicolumn{5}{|c|}{ SURIFE Stufe M } & max. 30 Punke & 29 Punkte \\
\hline \multicolumn{7}{|c|}{ Schwierigkeitsstufe E (hier sind sie Experte) } \\
\hline Datum & $\begin{array}{l}\text { Zeitdauer } \\
\text { (UE) }\end{array}$ & Sozialform & Thema & Schwierigkeit & $\begin{array}{l}\text { Maximale } \\
\text { Punkte }\end{array}$ & $\begin{array}{l}\text { Erreichite } \\
\text { Punkte }\end{array}$ \\
\hline $\begin{array}{l}10.11 .2007 \\
\text { bis } \\
17.11 .2007\end{array}$ & 8 & Einzelarbeit & $\begin{array}{l}\text { vom Schüler frei wählbar, bitte Thema mit der Lehrkraft vor } \\
\text { Beginn der Bearbeitung besprechen. } \\
\text { (Beispiel: Lernmodul für windows Registry mit Text, video } \\
\text { und Lernaufgaben erstellen) }\end{array}$ & Experte & 20 & 19 \\
\hline \multicolumn{5}{|c|}{ SUMIFE Stufe M } & max. 20 Punke & 19 Punkte \\
\hline
\end{tabular}

\begin{tabular}{|c|c|c|c|}
\hline \multicolumn{4}{|c|}{ Zusatzpunkte Z (rür eine besondere Mitarbeit) } \\
\hline Datum & Zeitdauer & Was habe ich gemacht & Zusatzpunkte \\
\hline 27.09 .2007 & 30 win. & $\begin{array}{l}\text { Der Schüler Bart Simpson hatte Probleme mit seinem PC, der ist dauernd abgestürzt. Tch habe ihm geholfen, den PC mit der } \\
\text { Wiederherstellungskonsole von Windows zu reparieren. }\end{array}$ & 2 \\
\hline 05.11 .2007 & 45 min. & $\begin{array}{l}\text { Die Schülerin Maggie Simpson hatte Probleme mit dem Umgang mit der Zone Alarm Firewall, weil sie letzte woche krank war. Ich } \\
\text { habe mit ihr gechattet und ihr grundlegende Fragen nochmals ausführlich beantwortet. Das Chatprotokoll wurde dem Betreuer der } \\
\text { Lernplattform am 05.11.2007 um 23:15 an die e-mail Adresse test@igmx, at gesendet. }\end{array}$ & 3 \\
\hline
\end{tabular}

Ihre Punkteanzahl:

\begin{tabular}{|l|l|}
\hline Stufe s (max. 100 Punkte möglich) & 87 Punkte \\
\hline Stufe M (max. 30 Punkte möglich) & 29Punkte \\
\hline Stufe (max. 20 Punkte möglich) & 19Punkte \\
\hline Stufe Z & 5 Punkte \\
\hline SUIFIFE & $\mathbf{1 4 0}$ Punkte \\
\hline
\end{tabular}

Abbildung 2: Zeit- und Contentfahrplan (Dreer, 2008)

\section{Notenskala:}

\begin{tabular}{|l|l|}
\hline Punkte & Ihre Note: \\
\hline$>=132$ & Sehr Gut \\
\hline $131-113$ & Gut \\
\hline $112-95$ & Befriedigend \\
\hline $94-76$ & Genügend \\
\hline$<=75$ & Nicht Genügend \\
\hline
\end{tabular}

Konzipiert wurde die Punkteverteilung so, dass ein Lernender für alle erfolgreich gelösten Lernaufgaben der Stufe S die Note «Befriedigend» erreichen kann, für alle Lernaufgaben der Stufe S und M die Note «Gut» und für alle Lernaufgaben der Stufe S, M und E die Note «Sehr Gut», vorausgesetzt man erreicht bei jeder Lernaufgabe eine hohe Punktezahl. Für den Einsatz im Unterricht könnte man zusätzlich noch den/die Schüler/in belohnen, der am Unterrichtsende die meisten Punkte erreicht hat (oder die drei besten Schüler/innen einer Lerngruppe), damit Schüler/innen, der beispielsweise schon alle Punkte für ein «Sehr Gut» erreicht haben, gegen Unterrichtsende noch immer motiviert sind, aktiv mitzuwirken und ev. auch bereits selbst erstellte Lernmodule mit neuen Ideen verbessern. 


\section{Fragestellung}

Es ist ein Ziel des Einsatzes von selbstgesteuerten Lernen mit einer elektronischen Lernumgebung, dass Lernvorgänge individueller gestaltet werden können. Um das zu untersuchen, wurde folgende Forschungsfrage aufgestellt:

Forschungsfrage: Wie kann selbstgesteuertes Lernen durch e-Learning unterstützt werden?

Um diese Forschungsfrage genauer zu spezifizieren, wurden Merkmale des selbstgesteuerten Lernens mit den Potenzialen von E-Learning in der mediendidaktischen Konzeption der Lernmodule des Projektes E-LEARNIT gegenüber gestellt:

\begin{tabular}{|c|c|}
\hline Merkmale selbstgesteuertes Lernen & $\begin{array}{l}\text { Potenziale von E-Learning in dieser } \\
\text { didaktischen Konzeption }\end{array}$ \\
\hline Zeit- und Ortsunabhängigkeit & Zeit- und Ortsunabhängigkeit \\
\hline $\begin{array}{l}\text { Wahlmöglichkeiten bei Themen und den damit } \\
\text { verbundenen Lernzielen }\end{array}$ & $\begin{array}{l}\text { Möglichkeit der Bearbeitung von unterschiedlichen } \\
\text { Lerninhalten von Lernenden zu einem Zeitpunkt }\end{array}$ \\
\hline Individuelles Lern- und Arbeitstempo & $\begin{array}{l}\text { Individuelles Lern- und Arbeitstempo durch } \\
\text { Hypertext-Navigation }\end{array}$ \\
\hline Hilfestellung durch den Lernbetreuer & $\begin{array}{l}\text { Hilfestellung (entweder durch den Lernbetreuer } \\
\text { durch Chat, e-mail, usw. oder durch Foren) }\end{array}$ \\
\hline Rückmeldung über den Lernerfolg & elektronische Rückmeldung \\
\hline
\end{tabular}

Tabelle 2: Selbstgesteuertes Lernen und E-Learning

Als weiteres wichtiges Merkmal selbstgesteuerten Lernens wird hier noch der Einsatz adäquater Lernstrategien erwähnt, dass jedoch in der elektronischen Lernumgebung nicht berücksichtigt wird, da es als Einführungsveranstaltung bzw. als laufende Unterstützung durch den Lehrenden individuell angeboten wird.

\section{Rahmenbedingungen}

Die Untersuchung wurde im Lehrgangsunterricht an 5 Lerngruppen (Lehrberufe IT-Technik und IT-Informatik) im Unterrichtsgegenstand EDV-Labor durchgeführt. Da nicht alle Berufsschüler sämtliche Fragebögen ausgefüllt haben, war die Anzahl der vollständig auswertbaren Datensätze bei 57 Probanden. In den Bereichen Vorbildung und Alter kann die Zielgruppe als heterogen eingestuft werden. Beim Pretest hat sich gezeigt, dass ein Grossteil der Lernenden mit dem Begriff «Feedback» nichts anfangen konnten («Was ist ein Feedback?»), weshalb im Fragebogen 
das Wort «Feedback» durch «Rückmeldung» ersetzt wurde. Die Schüler/innen waren einen überwiegend lehrerzentrierten Unterricht gewohnt. Während des Unterrichts mit E-Learning zeigten die Berufsschüler/innen eine engagierte Beteilung am Unterricht, die diese Methode als willkommene Abwechslung angenommen hat.

\section{Durchführung}

Zur Erfassung der Lernstrategien wurde der Fragebogen LIST (Lernstrategien im Studium) von Wild (2000) eingesetzt, da dieser sich auf unterschiedliche Theorien zum selbstgesteuerten Lernen, wie beispielsweise auf die Modelle von Weinstein und Pintrich, bezieht.

Der LIST-Fragebogen differenziert zwischen

- kognitiven Lernstrategien

- metakognitiven Lernstrategien und

- ressourcenbezogenen Lernstrategien.

Der LIST-Fragebogen gilt im deutschsprachigen Raum als das bekannteste Instrument zur Erfassung von Lernstrategien und wurde auch mehrfach empirisch belegt (Vgl. Spichinger, 2007). Bei der schriftlichen Befragung zur Erfassung der Fähigkeit der Selbststeuerung muss berücksichtigt werden, dass eher das theoretische Wissen über bestimmte Lernstrategien erfasst wird und dies nur wenig mit dem konkreten Einsatz bestimmter Lernstrategien bzw. mit bestimmten Kriterien von erhobenen Lernerfolgen korreliert (Leutner et. al, 2001; Carell, 2006). Um diesen Effekt zu minimieren wurde in dieser didaktischen Konzeption der Zeitraum zwischen der Bearbeitung der Lernmodule und der Datenerhebung möglichst kurz gehalten.

\section{Ergebnisse}

\section{Statistische Daten der befragten Personen}

55 Lernende waren männlich $(96,5 \%$, ist in diesem Berufsbereich nicht ungewöhnlich) und 2 Lernende waren weiblich (3,5\%). 3 Schüler (5,3\%) waren 16 Jahre alt, 7 Schüler (12,3\%) waren 17 Jahre alt, 11 Schüler (19,3\%) waren 18 Jahre alt, 12 Schüler $(21,1 \%)$ waren 19 Jahre alt und 24 Schüler $(42,1 \%)$ waren 20 Jahre alt oder älter. Ungefähr die Hälfte der Schüler befand sich in einem Jugendlichenalter.

Beim Lehrberuf haben 37 Schüler $(64,9 \%)$ den Berufsbereich IT-Technik angegeben und 20 Schüler $(35,1 \%)$ den Berufsbereich IT-Informatik. 


\section{Zuletzt absolvierte Schulform vor dem Besuch der Berufsschule}

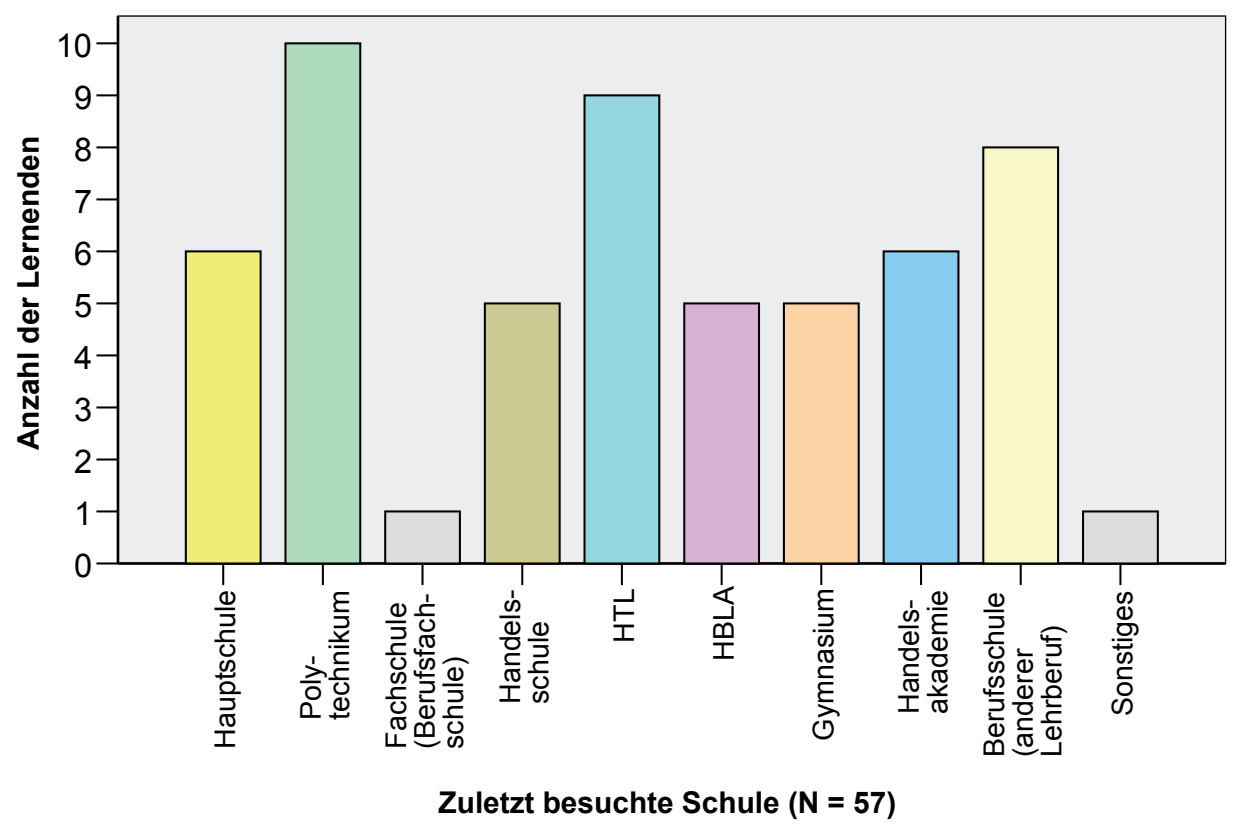

Abbildung 3: Ergebnisse - Zuletzt besuchte Schule

Im Bereich des Schulabschlusses haben 40 Schüler (70,2 \%) die Abschlussklasse bzw. eine oder mehrere Klassen der Vorbildung erfolgreich abgeschlossen. 6 Schüler (10,5\%) haben die Vorbildung nicht erfolgreich abgeschlossen und 11 Schüler $(19,3 \%)$ haben keine Angabe darüber getroffen. Ingesamt sind die Schüler im Bereich der Vorbildung als heterogen einzustufen.

Problematisch ist hier die Erhebung der Vorbildung insbesondere für den Bereich der Sonderschule bzw. ob die Hauptschule nicht erfolgreich abgeschlossen wurde, da das ein sensibler Bereich von persönlichen Daten ist und möglicherweise hier Lernende die Bereiche angegeben haben, die aus sozialer Sicht wünschenswert sind.

\section{Ergebnisse der Lernmodule}

Untersuchungsgegenstand waren folgende Lernmodule2:

- Apache Webserver unter Linux

- Samba Server unter Linux

- DNS Server unter Linux

siehe www.e-learnit.at.tf 
Beim Lernmodul Apache Webserver war die kürzeste Bearbeitungszeit 15 Minuten, das von zwei Lernenden innerhalb dieser Zeit bearbeitet wurde. Die längste Bearbeitungszeit war 130 Minuten, das von einem Lernenden innerhalb dieser Zeit bearbeitet wurde. Die durchschnittliche Bearbeitungszeit war 50 Minuten ( $M=$ 49,81 Minuten).

Beim Lernmodul Samba-Server war die kürzeste Bearbeitungszeit 10 Minuten, das von einem Lernenden innerhalb dieser Zeit bearbeitet wurde. Die längste Bearbeitungszeit war 142 Minuten, das von einem Lernenden innerhalb dieser Zeit bearbeitet wurde. Die durchschnittliche Bearbeitungszeit war 56 Minuten ( $M=56,35$ Minuten).

Beim Lernmodul DNS-Server war die kürzeste Bearbeitungszeit 15 Minuten, das von einem Lernenden innerhalb dieser Zeit bearbeitet wurde. Die längste Bearbeitungszeit war 250 Minuten, das von einem Lernenden innerhalb dieser Zeit bearbeitet wurde. Die durchschnittliche Bearbeitungszeit war 141 Minuten $(M=141,18$ Minuten).

Insgesamt gesehen liegen die durchschnittlichen Bearbeitungszeiten des ApacheWebservers und des Samba-Servers in einem ähnlichen Bereich (50 bzw. $56 \mathrm{Mi}-$ nuten). Beim DNS-Server war die Bearbeitungszeit insgesamt gesehen höher, da während des Unterrichts (im Gegensatz zu den anderen Lernmodulen) sehr viele technische Fragen aufgetaucht sind, weshalb hier die höhere Bearbeitungszeit möglicherweise darauf zurückzuführen ist.

Letztendlich haben aber alle Lernenden alle Server mit ausreichender Funktionalität in das Netzwerk hängen können, das auch für die spätere Berufspraxis ein wichtiges Ergebnis ist, da auch dort (praxisbezogen) Probleme bei Konfigurationen auftreten können, die gelöst werden müssen, wodurch auch die Lehr- und Lernziele in diesem Lernsetting erreicht wurden. Für den Lernerfolg wesentlich war das unterschiedliche Lerntempo, wo hier zwischen den Lernenden der Zeitfaktor sehr stark schwankte. Dies wurde auch schon in anderen Studien belegt und hiermit nochmals bestätigt.

Ein weiteres Ergebnis der empirischen Daten ist, das von keinem einzigen (!) Lernenden die Möglichkeit genutzt wurde, alle drei Lernmodule zu bearbeiten (obwohl einem Grossteil der Lernenden ausreichend Zeit hierfür zur Verfügung gestanden wäre) und die Lernenden explizit darauf hingewiesen wurden, das sie bei Interesse gerne alle Lernmodule bearbeiten dürfen (und ggf. auch Unterstützung erhalten). Dieses Ergebnis ist möglicherweise darauf zurückzuführen, dass die Lernenden grossteils extrinsisch motiviert waren. Die Ursache könnte aber auch an dem Betriebssystem Linux liegen, das für an Windows gewöhnte Benutzer nicht immer einfach zu bedienen ist.

Weiters wurde das Lernmodul «Samba-Server» (40 Lernende) gegenüber dem Lernmodul «DNS-Server» (17 Lernende) von den Lernenden bei der Wahlmöglichkeit bevorzugt. In manchen Lerngruppen wurde das Lernmodul DNS-Server über- 
haupt nicht bearbeitet. Dieses Ergebnis ist möglicherweise darauf zurückzuführen, das der Samba-Server in der Praxis stark verbreitet ist und die Lernenden eher einen Sinn darin gesehen haben, in ihrem Netzwerk einen Dateiserver mit Druckerfreigaben zu implementieren (Samba-Server) als einen Server, der die IP-Adressen in Domänennamen auflöst (DNS-Server).

\section{Individualisierung bzw. selbstgesteuertes Lernen}

Reihenfolge der Bearbeitung der Inhalte und Lernzeit

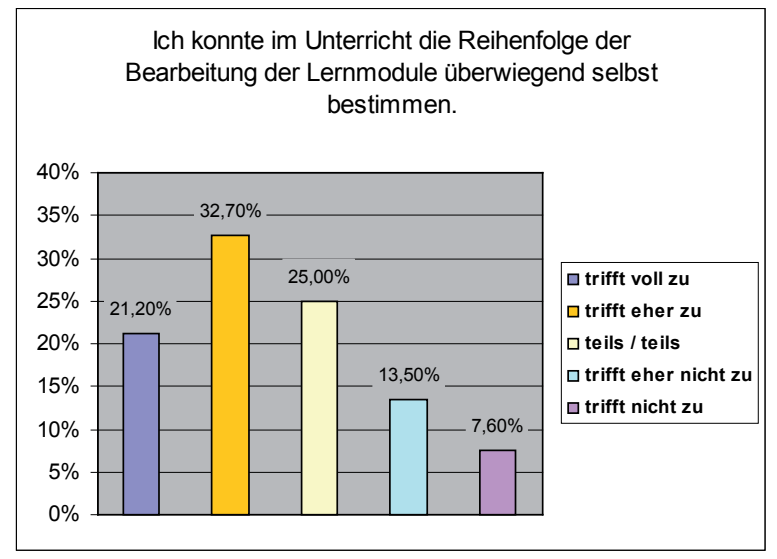

Abbildung 4: Ergebnisse: Reihenfolge der Bearbeitung der Lernmodule
Im Unterricht konnte ich zu einem Zeitpunkt selbst bestimmen, welchen Abschnitt ich gerade bearbeite.

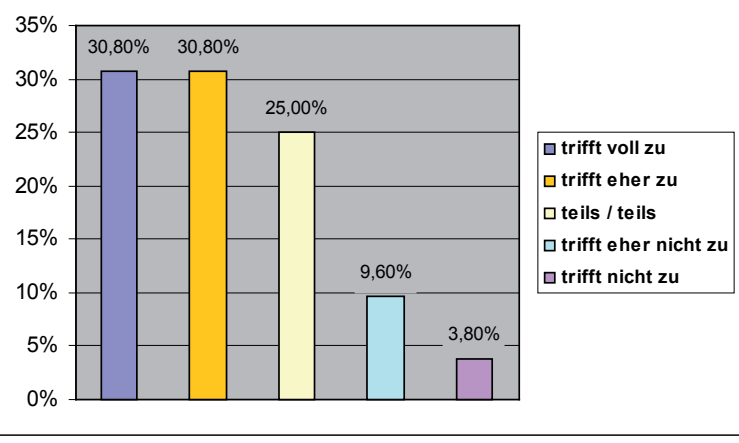

Abbildung 5: Ergebnisse: Zeitpunkt / Abschnitt

$78,9 \%$ der Lernenden konnten im Unterricht die Reihenfolge der Bearbeitung der Lernmodule (Abb. 4) selbst bestimmen (21,2\% der Lernenden - trifft voll zu, 32,7 $\%$ der Lernenden - trifft eher zu, $25 \%$ der Lernenden - teils / teils). 21,1 \% der Lernenden waren der Ansicht, dass sie die Reihenfolge nicht selbst bestimmen konnten (13,5\% der Lernenden - trifft eher nicht zu, 7,6 \% der Lernenden - trifft nicht zu).

$86,6 \%$ der Lernenden konnten im Unterricht zu einem Zeitpunkt (Abb. 4) selbst bestimmen, welchen Abschnitt sie gerade bearbeiten (30,8\% der Lernenden - trifft voll zu, 30,8 \% der Lernenden - trifft eher zu, $25 \%$ der Lernenden - teils / teils). $13,4 \%$ der Lernenden waren der Ansicht, dass sie das eher nicht selbst bestimmen konnten $(9,6 \%$ der Lernenden - trifft eher nicht zu, 3,8 \% der Lernenden - trifft nicht zu). Dieses Ergebnis ist positiv zu beurteilen. 


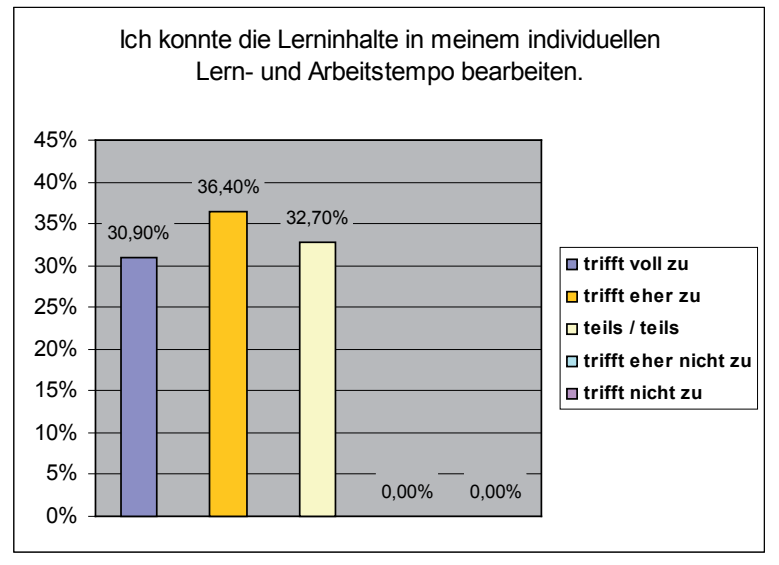

Abbildung 6: Ergebnisse: Individuelles Lern- und Arbeitstempo

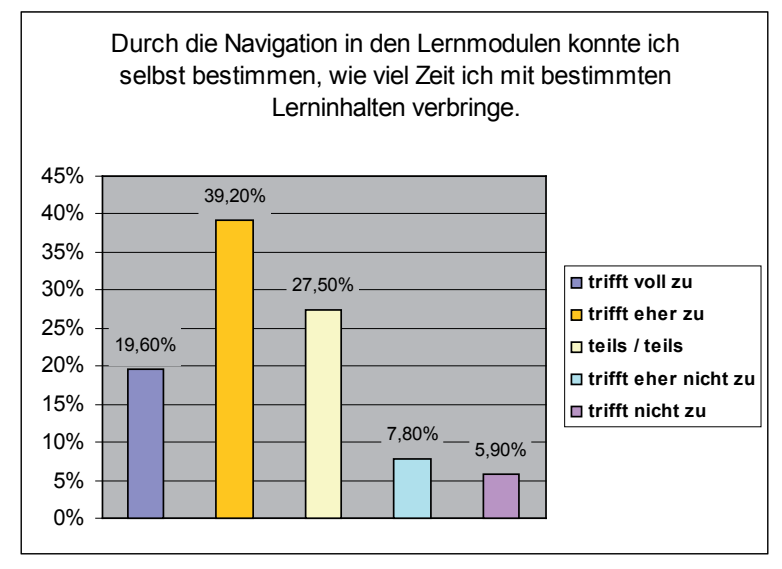

Abbildung 7: Ergebnisse: Navigation und Zeit

Dieses Ergebnis ist positiv zu beurteilen, da gerade das individuelle Lern- und Arbeitstempo ein kritischer Erfolgsfaktor für die didaktische Konzeption ist.

\section{Schwerpunkte}

In der didaktischen Konzeption hatten die Lernenden folgende Möglichkeiten:

- Wahlmöglichkeit bei den Lernmodulen

- Weglassen eines Lernmodules

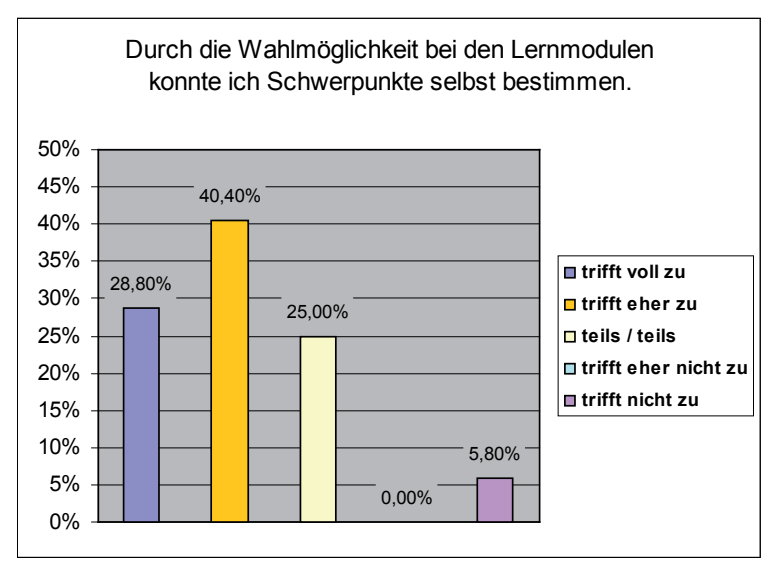

Abbildung 8: Ergebnisse: Wahlmöglichkeit

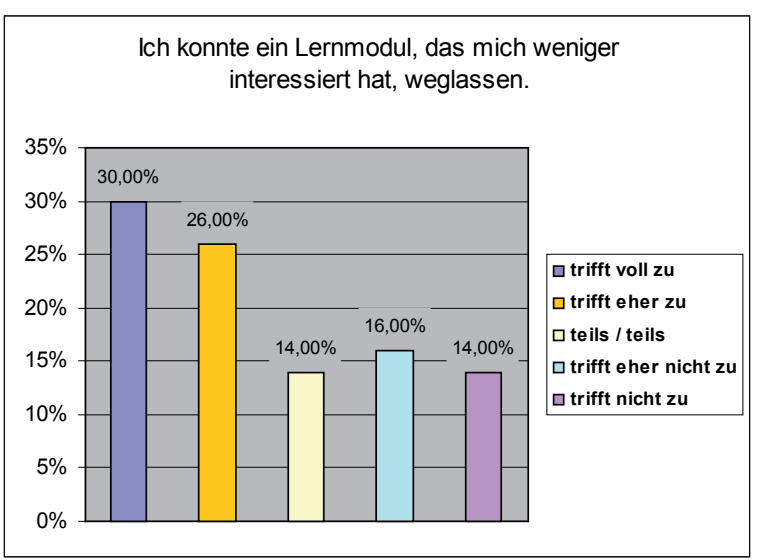

Abbildung 9: Ergebnisse: Lernmodul weglassen

94,2\% der Lernenden gaben an, durch die Wahlmöglichkeit Schwerpunkte (Abb. 8) selbst bestimmen zu können (28,8 \% der Lernenden - trifft voll zu, 40,4 \% der Lernenden - trifft eher zu, $25 \%$ der Lernenden - teils / teils). 5,8\% der Lernenden waren der Ansicht, dass sie keine Wahlmöglichkeit hatten bzw. Schwer- 
punkte nicht selbst bestimmen konnten (5,8 \% der Lernenden - trifft nicht zu). Dieses Ergebnis ist durch die hohe Zustimmung der Lernenden als positiv zu beurteilen.

$70 \%$ der Lernenden gaben an, ein Lernmodul, das weniger interessant ist (Abb. 9), weglassen zu können (30 \% der Lernenden - trifft voll zu, $26 \%$ der Lernenden - trifft eher zu, $14 \%$ der Lernenden - teils / teils). $30 \%$ der Lernenden waren der Ansicht, dass das nicht möglich war (16\% der Lernenden - trifft eher nicht zu, $14 \%$ der Lernenden - trifft nicht zu).

Dass diese Möglichkeiten vorhanden waren, wurde auch durch die vorliegenden Untersuchungsergebnisse bestätigt.

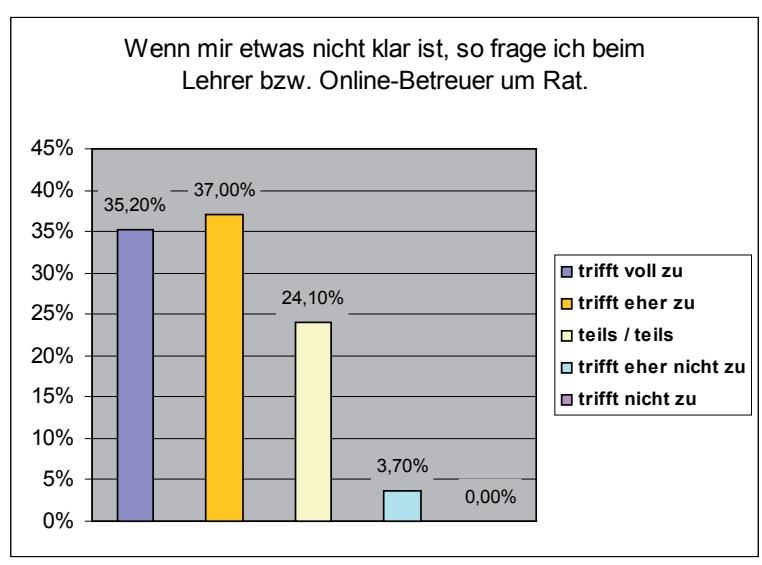

Abbildung 10: Ergebnisse: Lehrer/in bei Unklarheiten um Rat fragen

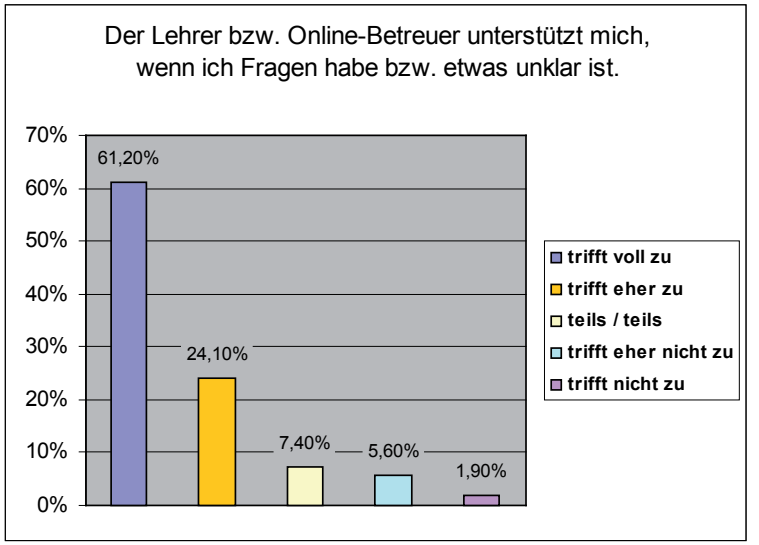

Abbildung 11: Ergebnisse: Lehrer/in Unterstützung

\section{Hilfestellung und Unterstützung}

Die Hilfestellung und Unterstützung des Lehrenden sind auch kritische Erfolgsfaktoren für den Einsatz des selbstgesteuerten Lernens.

Aufgrund der vorliegenden Ergebnisse ist auch dieser kritische Erfolgsfaktor im Bereich des selbstgesteuerten Lernens mit E-Learning in dieser didaktischen Konzeption erfüllt und die Unterstützung und Hilfestellung seitens der Lehrkraft bzw. des Online-Betreuers ist durch die hohe Zustimmung (jeweils über $90 \%$ ) als positiv zu beurteilen. 


\section{LIKERT-Skala mit den besten bzw. schlechtesten Lernstrategien}

In folgender LIKERT-Skala wurde der Mittelwert aller Lernenden in den einzelnen Kategorien ermittelt, der Mittelwert mit den 25\% der Lernenden mit den besten Lernstrategien (Lerngruppe B) und der Mittelwert der 25\% der Lernenden mit den schlechtesten Lernstrategien (Lerngruppe S). Sämtliche Mittelwerte beziehen sich auf die eingesetzten Lernstrategien während der Bearbeitung der elektronischen Lernmodule.

\begin{tabular}{|c|c|c|c|}
\hline & Mittelwert & Mittelwert & Mittelwert \\
\hline $\begin{array}{l}\text { LIKERT-Skala } \\
\text { Lernstrategien }\end{array}$ & $\begin{array}{l}\text { aller } \\
\text { Lernenden } \\
(\mathrm{N}=57)\end{array}$ & $\begin{array}{l}25 \% \text { der Lernenden } \\
\text { mit den besten } \\
\text { Lernstrategien } \\
\text { (unteres Quartil) } \\
\text { (N=15, Lerngruppe B) }\end{array}$ & $\begin{array}{l}25 \% \text { der Lernenden } \\
\text { mit den schlechtesten } \\
\text { Lernstrategien } \\
\text { (oberes Quartil) } \\
\text { (N=15, Lerngruppe S) }\end{array}$ \\
\hline A) Organisation & 1,71 & 1,40 & 1,98 \\
\hline B) Anstrengung & 2,26 & 1,86 & 2,48 \\
\hline C) Lernumgebung & 2,11 & 1,78 & 2,53 \\
\hline D) Planung & 2,54 & 2,03 & 3,19 \\
\hline E) Kontrolle & 2,79 & 2,20 & 3,34 \\
\hline F) Selbststeuerung & 2,48 & 2,10 & 2,86 \\
\hline G) Konzentration & 2,89 & 2,51 & 3,50 \\
\hline H) Zeitmanagement & 3,09 & 2,76 & 3,25 \\
\hline I) Weiterführende Informationen & 2,42 & 1,75 & 3,07 \\
\hline J) Kritisches Prüfen & 3,05 & 2,78 & 3,40 \\
\hline K) Wiederholen & 2,79 & 2,35 & 3,09 \\
\hline L) Lernen im Team & 2,37 & 1,93 & 2,98 \\
\hline M) Individueller Schwerpunkt & 2,38 & 1,74 & 3,27 \\
\hline N) Online-Betreuer & 1,80 & 1,46 & 2,17 \\
\hline Mittelwert Lernstrategien: & 2,48 & 2,05 & 2,94 \\
\hline
\end{tabular}

Tabelle 3: Ergebnisse: LIKERT-Skala Lernstrategien 
Vergleicht man die Gruppen der Lernenden mit den besten bzw. den schlechtesten Lernstrategien, so sind in allen Bereichen der Lernstrategien Unterschiede festzustellen. Die Lerngruppe mit den besten Lernstrategien (Lerngruppe B) unterscheidet sich immer bzw. ist immer besser einzustufen als die Lerngruppe mit den schlechtesten Lernstrategien (Lerngruppe S).

Es wurden von beiden Lerngruppen gute Ergebnisse im Bereich der Organisation (A) und bei der Beurteilung des Online-Betreuers (N) erzielt. Hier liegen die Mittelwerte im Bereich der Organisation (A) zwischen $M=1,40$ (Lerngruppe $B$ ) und $M=1,98$ (Lerngruppe S). Im Bereich der Beurteilung des Online-Betreuers bzw. der Lehrkraft $(N)$ liegen die Mittelwerte zwischen $M=1,46$ (Lerngruppe $B$ ) und $M$ $=2,17$ (Lerngruppe $\mathrm{S}$ ). Das die Betreuung der Lernenden gut sein muss ist auch ein kritischer Erfolgsfaktor, damit selbstgesteuertes Lernen überhaupt stattfinden kann, das in diesem Lernsetting auch so war und durch diese Auswertung bestätigt werden konnte.

Wie man im nachfolgenden Netzdiagramm erkennen kann, ist die Lerngruppe B in allen Kategorien besser als die Lerngruppe S. Die Mittelwerte der Lernstrategien aller Lernenden liegen in allen Kategorien im Mittelfeld. 


\section{Lernstrategien}

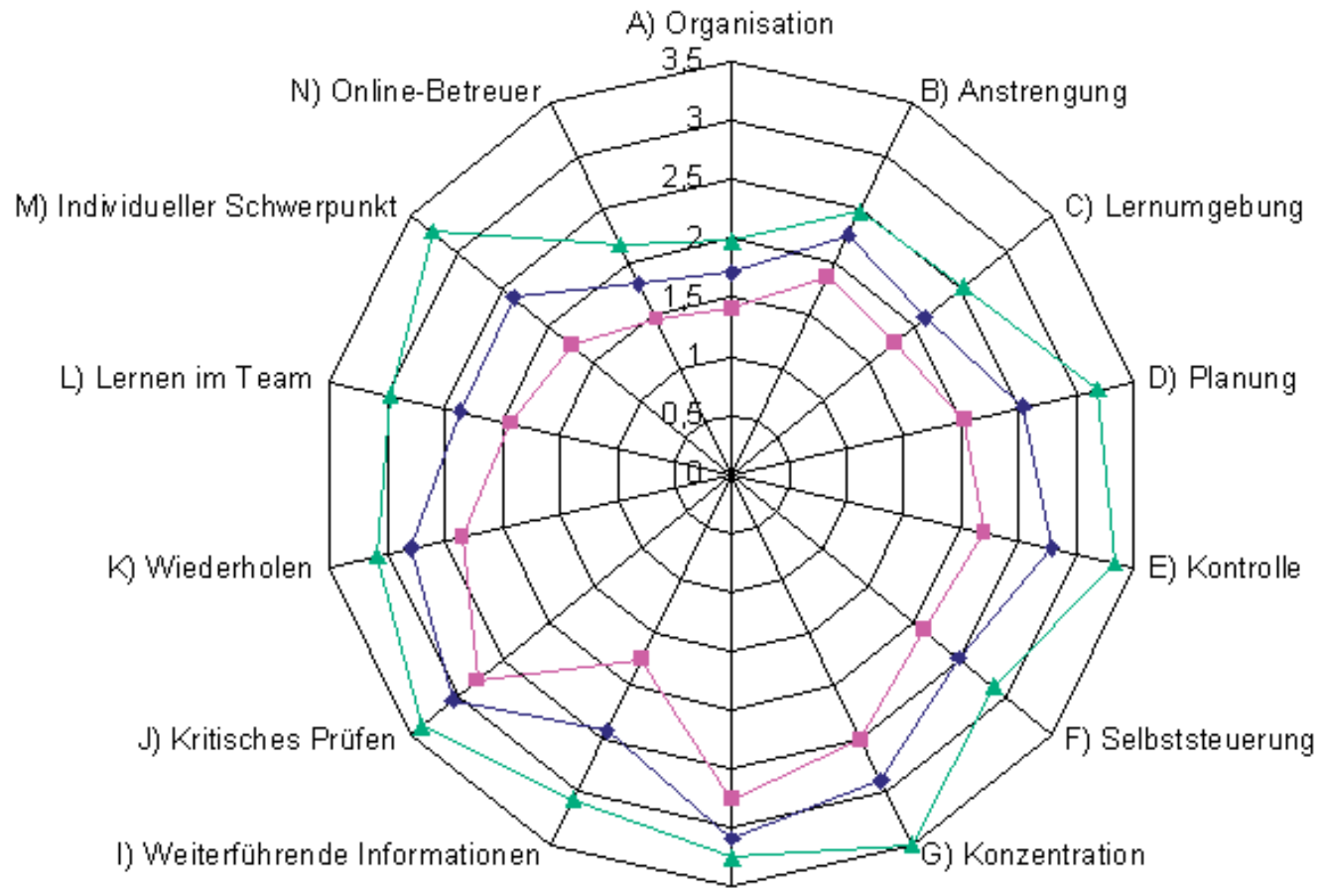

H) Zeitmanagement

- - alle Lernenden ( $\mathrm{N}=57$ )

$=-25 \%$ mit den besten Lern strate gien

$-\downarrow-25 \%$ mit den schlechtesten Lernstrategien

Abbildung 12: Ergebnisse: LIKERT-Skala Lernstrategien gute bzw. schlechte Lerngruppen und alle Lernenden

Insgesamt gesehen sind die eingesetzten Lernstrategien der Lernenden im Bereich gut bis mittelmässig anzusiedeln, wobei hier in einigen Bereichen - insbesondere für die Lernstrategien H) Zeitmanagement $M=3,09$ und J) Kritisches Prüfen $M=3,05$ - geringere Mittelwerte erwartet wurden. Gerade durch den Einsatz des Zeit- und Contentfahrplanes im Rahmen der didaktischen Konzeption der Lernumgebung wurden hier bessere Ergebnisse von den Lernenden erwartet. Im Rahmen der Wahlmöglichkeit bei den Lernmodulen wurde auch erwartet, dass die Lernenden die Lerninhalte kritischer (insbesondere vor und während der Bearbeitung) überprüfen.

Bei der Lerngruppe S (schlechtesten Lernstrategien) sind einige Bereiche noch verbesserungswürdig, insbesondere die Planung, Kontrolle, Konzentration, Zeitmanagement, weiterführende Informationen, kritisches Prüfen und Wiederholen (alle Bereiche, deren Mittelwert grösser als 3.0 ist). 
Im Bereich des individuellen Schwerpunktes wurde diese Kategorie auch von den Lernenden sehr individuell beurteilt: die Lerngruppe B (besten Lernstrategien) hat mit $M=1,74$ ein gutes Ergebnis erzielt, während die Lerngruppe $S$ (schlechtesten Lernstrategien) hier mit $M=3,27$ die Individualisierung als verbesserungswürdig erachtet.

Betrachtet man die Ergebnisse insgesamt, gibt es bei den eingesetzten Lernstrategien, besonders bei Lernenden, die ihre eingesetzten Lernstrategien schlechter beurteilen als andere Lernende, noch ein Verbesserungspotenzial.

\section{Selbstgesteuertes Lernen durch E-Learning}

Die Untersuchung zeigt, dass selbstgesteuertes Lernen durch E-Learning in einem bestimmten Ausmass unterstützt werden kann. Eine vollständige Selbststeuerung wird im Einsatzbereich der Schulen niemals stattfinden, da bestimmte Lerninhalte, Lernzeiten und Lernorte durch den Lehrplan bzw. Stundenplan festgelegt sind.

Forschungsfrage: Wie kann selbstgesteuertes Lernen durch E-Learning unterstützt werden?

Durch den Einsatz von E-Learning in dieser didaktischen Konzeption wurde während des Unterrichts insbesondere das individuelle Lern- und Arbeitstempo, die Möglichkeit der Bearbeitung von unterschiedlichen Lerninhalten von Lernenden zu einem Zeitpunkt, die Hilfestellung durch den Lernbetreuer und eine individuelle (persönliche) bzw. elektronische Rückmeldung über den Lernerfolg ermöglicht. Wie in folgender Abbildung ersichtlich wird, liegen die Kriterien (die aufsteigend sortiert wurden), damit überhaupt selbstgesteuerte Lernen stattfindet, nicht im Bereich der absoluten Autonomie sondern eher im Bereich der selbstgesteuerten Lernens (ca. $60-80 \%$ ) und darüber und keine Kriterien befinden sich auch nur annähernd im Bereich der Fremdsteuerung (0 \%), weshalb diese Forschungsfrage bestätigt werden kann. 


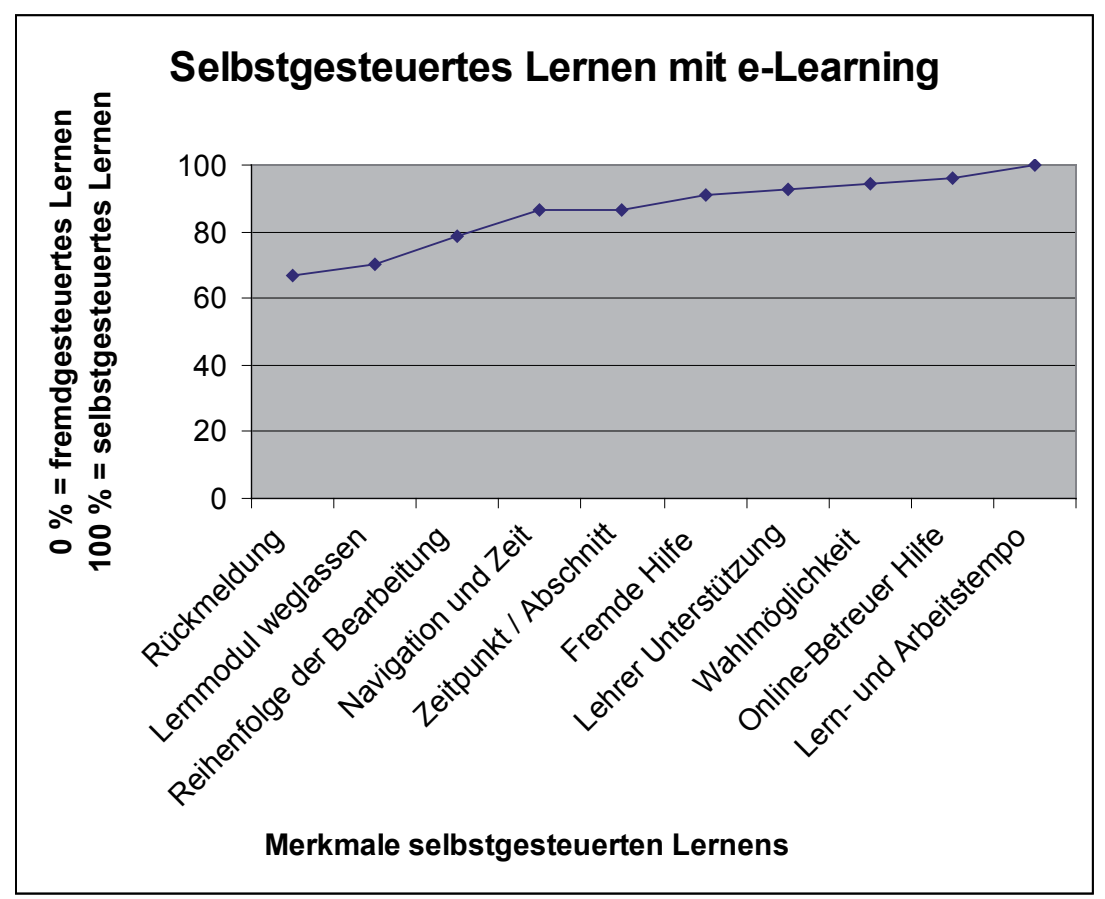

Abbildung 13: Ergebnisse: Selbstgesteuertes Lernen mit E-Learning

Durch die neuen Freiräume, die durch den Einsatz von E-Learning im Unterricht entstehen, können diese zur individuellen Förderung und den damit verbundenen Lernstrategietraining von besonders leistungsschwachen Schülern verwendet werden, das im herkömmlichen Unterricht nicht in diesem Ausmass möglich ist.

\section{Fazit}

Im vorliegenden Beitrag wird selbstgesteuertes Lernen anhand vieler unterschiedlicher Faktoren bestimmt und es wird im Kontext Schule eine vollständige Selbststeuerung nur unter bestimmten Vorraussetzungen stattfinden, da bestimmte Lerninhalte, Lernzeiten und Lernorte durch den Lehr- bzw. Stundenplan festgelegt sind. Ein wichtiges Ziel dieses Beitrages war ein Bildungsanliegen durch den Einsatz von E-Learning zu lösen, das gelungen ist, wie die vorliegenden Ergebnisse auch bestätigt haben. Durch das heterogene Vorwissen der Lernenden bzw. durch die teilweise fehlende Berufspraxis sind für bestimmte, von der Berufsschule zu vermittelnde Lerninhalte, unter den Lernenden - vom Anfänger bis zum Experten - alles im Unterricht vertreten, das sich auch auf das Lerntempo während der Bearbeitung von Lernaufgaben ausgewirkt hat. Weiters werden durch den Einsatz von E-Learning Medienkompetenzen und die im Lehrplan gesetzlich geforderte Methodenvielfalt während des Unterrichts noch zusätzlich gefördert. Durch diese 
didaktische Konzeption hatten die Lernenden die Möglichkeit, sich einen individuellen Schwerpunkt im Unterricht zu setzen bzw. einen Themenbereich, der weniger interessant war, weglassen zu können. Durch die Potenziale von E-Learning konnten durch das zeit- und ortsunabhängige Lernen und die individuelle Bestimmung der Lerngeschwindigkeit die Lernprozesse durch den Lernenden individueller gestaltet werden. Es sind dem Lernenden verschiedene Kommunikationsmöglichkeiten angeboten worden bzw. elektronische Lernmaterialien zur Verfügung gestellt worden bzw. hatte der Lernende die Möglichkeit, eine Lernerfolgskontrolle selbst durchführen zu können.

Diese vorliegenden Untersuchungsergebnisse leisten einen Beitrag dazu, inwieweit sich die Potenziale von E-Learning mit selbstgesteuertem Lernen in Einklang bringen lassen bzw. inwieweit auch selbstgesteuertes Lernen in einer medialen Lernumgebung von den Lernenden in berufsbildenden Schulen eingesetzt werden kann. Sollten sich diese Ergebnisse in weiterführenden Studien nochmals bestätigen lassen, könnten sich daraus noch weitere Ansätze für die Verbesserung des selbstgesteuerten Lernens gerade im Kontext Schule (wo das selbstgesteuerte Lernen sehr gering angesiedelt ist) aufzeigen lassen. 


\section{Literatur}

Ablard, Karen E.; Lipschultz, Rachelle E. «Self-regulated learning in high-achieving students. Relations to advanced reasoning, achievement goals and gender.» Journal of Educational Psychology 90 (1998): 94-101.

Aeppli Jürg. Selbstgesteuertes Lernen von Studierenden in einem Blended-Learning-Arrangement: Lernstil-Typen, Lernerfolg und Nutzung von webbasierten Lerneinheiten. Dissertation, Universität Zürich: Zentralstelle der Studentenschaft der Universität Zürich, 2005.

Astleitner Hermann; Hofmann Franz; Ziegler Karin. Selbstreguliertes Lernen und Internet. Theoretische und empirische Grundlagen von Qualitätssicherungsmassnahmen beim ELearning. Frankfurt am Main: Peter Lang GmbH, 2003.

Baumert Jürgen Pisa 2000. Ein differenzierter Blick auf die Länder der Bundesrepublik Deutschland. Deutsches PISA-Konsortium, 2003.

BMBWK. Nutzung der Lernplattform moodle; Angebot des bmbwk, Pilotprojekt „edumoodle.at. Bundesministerium für Bildung, Wissenschaft und Kultur. Erlass. (2006): http://www.edumoodle.at/moodle/file.php/1/edumoodle_erlass_und_information.pdf (20.04.2008).

Boekaerts Monique. "Self-regulated learning. Where we are today.»International Journal of Educational Research 31 (1999): 445-457.

Borkowski, John G.; Chan, Lorna K. S.; Muthukrishna, Nithi. «A process-oriented model of metacognition: Links between motivation and executive functioning.» Schraw Gregory; Impara James C. (Eds.), Issues in the measurement of metacognition. Lincoln: The University of Nebraska Press, 2000.

Bräu Karin. «Individualisierung des Lernens - Zum Lehrerhandeln bei der Bewältigung eines Balanceproblems.» Bräu K.; Schwerdt U. (Hrsg.): Heterogenität als Chance. Vom produktiven Umgang mit Gleichheit und Differenz in der Schule, LIT Verlag Münster, 2005.

Carell Angela. Selbststeuerung und Partizipation beim computerunterstützten kollaborativen Lernen. Eine Analyse im Kontext hochschulischer Lernprozesse. Waxmann Verlag (Münster/New York/Berlin/München) Band 37 (2006).

Dreer, Silvia. e-Learning als Ansatz einer individualisierten Lernstrategie an einer Berufsschule für Informationstechnik. Dissertation, Duisburg-Essen: Universität, 2008.

Dreyer Jasmin. «Die Förderung zur Befähigung zum selbst gesteuerten Lernen im Unterricht an berufsbildenden Schulen über die Vermittlung von Lernstrategien.» Diplomarbeit. Johannes Gutenberg-Universität Mainz, 2007.

Dumke, Dieter; Wolff-Kollmar, Sabine. "Lernstrategien in der Beurteilung von Lehrern und Schülern.»Psychologie in Erziehung und Unterricht 44 (1997): 165-175.

Friedrich, Helmut F.; Mandl Heinz. "Analyse und Förderung selbstgesteuerten Lernens.» Weinert, F. E. (Hrsg.): Enzyklopädie der Psychologie: Psychologie d. Erwachsenenbildung, Göttingen, (1997): 237-276.

Gudjons Herbert. «Pädagogik - Einführung in das Thema Selbstgesteuertes Lernen der Schüler: Fahren ohne Führerschein?» (2003): http://www.beltz.de/paedagogik/ heft200305/n_02_02.html (20.04.2008).

Hamman, Douglas; Berthelot, Joelle; Saia, Jodi; Crowley, Ellen. «Teachers' coaching of learning and its relation to students' strategic learning.» Journal of Educational psychology 92 (2000): 342-348.

Hasenpflug Beate; Eckelmann Astrid; Hahnke Marion; Markstein Ines; Schulze Carmen; Walther Kerstin. E-Learning und Lernsoftware im Fremdsprachenunterricht unter beson- 
derer Berücksichtigung der kaufmännischen Berufsschule. Sächsisches Staatsministerium für Kultus (Hrsg.): Stoba Druck: Sachsen (2004): http://www.sachsen-macht-schule. de/sabw/hr_elearning.pdf (20.04.2008).

Kerres, Michael; Petschenka Anke. «Didaktische Konzeption des Online-Lernens für Weiterbildung.» Burkhard Lehmann \& Egon Bloh (Hrsg.) Online Pädagogik. Grundlagen der Berufs- und Erwachsenenbildung. Baltmannsweiler: Schneider Verlag. Band 29 (2002): 240-256.

Leutner, Detlev; Barthel, Anke; Schreiber, Beate. «Studierende können lernen, sich selbst zum Lernen zu motivieren: Ein Trainingsexperiment.» Zeitschrift für Pädagogische Psychologie 15(3,4) (2001): 155-167.

Metzger Christoph; Nüesch, Charlotte; Zeder, Andrea; Jabornegg, Daniel. Förderung und Prüfung von Lernkompetenzen in der kaufmännischen Grundbildung. St. Gallen: Institut für Wirtschaftspädagogik, 2005.

Metzger, Christoph. Wie lerne ich? WLI-Schule. Eine Anleitung zum erfolgreichen Lernen für Mittelschulen und Berufsschulen. Aarau (6. Aufl.), 2006.

Perry, Nancy E. "Young childrens's self-regulated learning and contexts that support it.» Journal of Educational Psychologie 90 (1998): 715-729.

Pintrich Paul R. "The role of goal orientation in self-regulated learning.» Boekaerts Monique; Pintrich Paul R.; Zeidner Moshe (Eds.), Handbook of self-regulation. San Diego, CA: Academic Press (2000): 451-502.

Salchegger, Martin. Unterstützung selbstregulierten Lernens im Unterricht. Massnahmen zur Förderung selbstregulierten Lernens an berufsbildenen mittleren und höheren Schulen (BMHS). Diplomarbeit, Fachhochschule Hagenberg, 2005.

Schraw, Gregory. «Promoting general metacognitive awareness.» Instructional Science 26 (1998): 113-125.

Siebert, Horst. Selbstgesteuertes Lernen und Lernberatung. Neuwied: Luchterhand, 2001.

Spichiger Katja. «Wahl der Lernstrategie unter Studierenden, eine Altersfrage?» Methodenpropädeutikum I der Angewandten Psychologie \& der Psychologischen Methodenlehre. Zürich: Universität, Psychologisches Institut, 2007.

Stenger Fabian. Selbstreguliertes Lernen im Modellversuch KoLA: Empirische Analyse zu den Lernvoraussetzungen und insbesondere dem selbstbezogenen Vertrauen von Schülern berufsbildender Schulen. Diplomarbeit, Johannes Gutenberg-Universität, Mainz, 2007.

Tiaden Corinne. Selbstreguliertes Lernen in der Berufsbildung: Lernstrategien messen und fördern. Diss., Universität Basel (2006): http://pages.unibas.ch/diss/2006/DissB_7762. pdf (20.04.2008).

van Den Hurk Marianne. "The relation between self-regulated strategies and individual study time, prepared participation and achievement in a problem-based curriculum.» Active Learning in Higher Education 7 (2006): 155-169.

Weinstein, Claire E. «Strategic learning / strategic teaching. In Pintrich Paul R.; Brown Donald R.; Weinstein Claire E. (Eds.), Student motivation, cognition, and learning.» McKeachie Wilbert J. Hillsdale. NY: Erlbaum (1994): 257-273.

Wild, Klaus-Peter. Lernstrategien im Studium. Strukturen und Bedingungen. Münster: Waxmann. (2000): 〈http://www-campus.uni-r.de/edu1/index.php?option=content\&task=vie w\&id=28\&ltemid=109, (20.04.2008).

Wuttke, Eveline. Motivation und Lernstrategien in einer selbstorganisationsoffenen Lernumgebung. Eine empirische Untersuchung bei angehenden Industriekaufleuten. Disseration, (Peter Lang) Frankfurt, New York, Toronto, 1999. 
Zimmerman, Barry J.; Schunk, Dale H. «Reflections on theories of self-regulated learning and academic achievement.» In Barry J. Zimmerman \& Dale H. Schunk (Eds.), Self-regulated learning and achievement: Theoretical perspectives. Mahwah: Erlbaum (2001): 289-307.

Zimmermann, Barry J. «Attaining Self-Regulation: a social cognitive perspective.» Boekaerts, Monique; Pintrich, Paul R.; Zeidner, Moshe (Eds.), Handbook of Self-Regulation. Academic Press., 2000. 